\title{
Raptor Network Video Coding
}

\author{
Nikolaos Thomos \\ Ecole Polytechnique Fédérale de Lausanne \\ (EPFL) \\ Signal Processing Institute - LTS4 \\ $\mathrm{CH}-1015$ Lausanne, Switzerland \\ nikolaos.thomos@epfl.ch
}

\author{
Pascal Frossard \\ Ecole Polytechnique Fédérale de Lausanne \\ (EPFL) \\ Signal Processing Institute - LTS4 \\ CH-1015 Lausanne, Switzerland \\ pascal.frossard@epfl.ch
}

\begin{abstract}
In this paper, we consider the problem of streaming video information on lossy packet networks with path and source diversity, such as wireless mesh networks or more generally overlay architectures. We propose to distribute the video information between several streaming servers and to implement network coding in the forwarding peers in order to increase the quality of the video decoded by the streaming client. A network coding algorithm based on Raptor codes permits to combine video packets in the network nodes and to increase the symbol diversity in the network. Coupled with the path diversity in the overlay network, this solution is shown to offer a significant gain in video quality compared to baseline streaming solutions when loss rate becomes important or the network diversity is reduced.
\end{abstract}

\section{Categories and Subject Descriptors}

H.4.3 [Information Systems Applications]: Communications applications - Computer conferencing, teleconferencing, and videoconferencing

\section{General Terms}

Design, Performance.

\section{Keywords}

Network coding, overlay networks, error resiliency

\section{INTRODUCTION}

Today's network architectures offer increasing diversity to multimedia communication applications, as they often provide several streaming paths to the receivers as well as multiple streaming sources. Peer-to-peer systems, wireless mesh networks, or overlay infrastructures represent such architectures where aggregation of network and computing resources permit to improve the quality of service offered to the streaming client. Network diversity compensates for the

Permission to make digital or hard copies of all or part of this work for personal or classroom use is granted without fee provided that copies are not made or distributed for profit or commercial advantage and that copies bear this notice and the full citation on the first page. To copy otherwise, to republish, to post on servers or to redistribute to lists, requires prior specific permission and/or a fee.

MV'07, September 28, 2007, Augsburg, Bavaria, Germany. Copyright 2007 ACM 978-1-59593-779-7/07/0009 ...\$5.00 lack of quality of service guarantee in these dynamic and best effort networking environments.

Network diversity can be exploited by streaming video information from multiple sources or via multiple network paths, in order to increase the overall throughput and the robustness to packet loss. At the same time, the streaming application could also take benefit of the computing capabilities of network nodes that should not act as simple relay nodes, but rather participate to improving the performance of the streaming application. Network coding [3] has been proposed recently as a promising framework to improve network throughput by performing packet coding operations in the network nodes. Network coding is generally considered in multicast scenarios and only a few studies have considered the use of network coding for video streaming in heterogeneous networks. We propose in this paper to build on the benefits of rateless codes in order to facilitate the deployment of network coding strategies in overlay or mesh architectures for improved video quality.

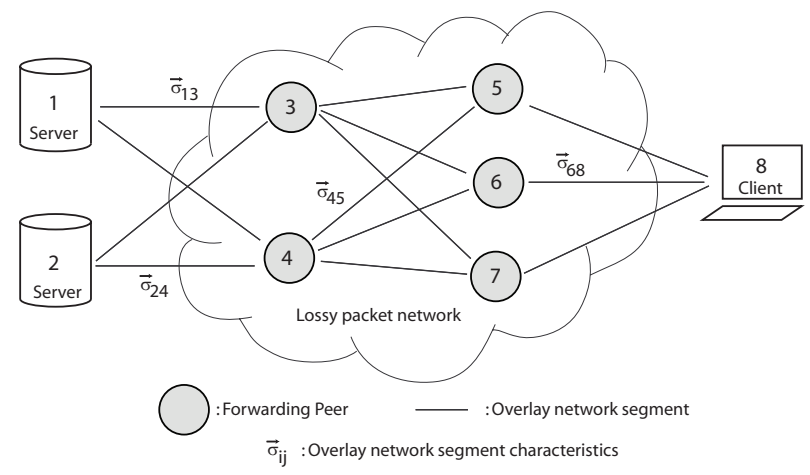

Figure 1: Streaming with network diversity. Multiple servers transmit media information to a client via a set of forwarding peers arranged in stages or hops.

In particular, we consider the problem of streaming of video information in mesh or overlay packet networks with lossy channels, as illustrated in Figure 1. A streaming client is connected to multiple sources through several forwarding peers that form a streaming overlay infrastructure. The forwarding peers are arranged in successive stages or hops, depending on the distance to the sources (computed as the number of overlay nodes traversed by information flow). An overlay network with $s$ sources, $x$ nodes in the first hop, $y$ 
nodes in the second hop, and $r$ client is denoted in this paper as a $s-x-y-r$ architecture. Each segment between nodes $i$ and $j$ in this infrastructure is characterized by a set of parameters $\vec{\sigma}_{i j}=\left[r_{i j}, \pi_{i j}, \alpha_{i j}\right]$, which respectively represent the available bandwidth, the packet loss ratio and the average length of bursts of errors. We propose to exploit the diversity offered by the network in order to improve the performance of the streaming applications. In particular, we encode video information and distribute redundant packets among multiple streaming sources. In addition, the forwarding peers implement network coding strategies based on Raptor codes [14]. These peers gather packets from ancestors peers, and re-encode the video packets in order to maintain high the symbol diversity in the network. Coupled with the use of multiple streaming paths, such a strategy becomes quite efficient when loss rates are particularly important with the network, as it is typically the case in wireless infrastructures. We apply the Raptor-based network coding algorithm to video streaming, and we show that it leads to better video quality when compared to state-of-the-art solutions.

The paper is organized as follows. Section II presents the network coding algorithm, based on Raptor codes. Section III extends the network coding scheme to video streaming applications. Section IV illustrates the performance of the Raptor-based network video coding scheme for several network scenarios, and shows that the proposed strategy is particularly beneficial when losses are quite important, or when the number of nodes per hops is limited. Section $\mathrm{V}$ finally describes the related work.

\section{RAPTOR NETWORK CODING}

\subsection{Overview of Raptor codes}

Channel coding in multimedia systems is generally based on Reed-Solomon (RS) codes to cope with packet erasures. However, their usage is limited in practice to small coding blocks due to their quadratic decoding complexity. Due to such limitations, fountain and Raptor codes have received a lot of interest recently since they provide increased performance, with a reduced complexity. They have been proposed respectively for peer-to-peer multimedia delivery [16] and wireless broadcast systems [13]. Raptor codes are also quite interesting for network coding, as they allow for simple symbol combinations (XOR) in the network, and they have interesting rateless properties that render them particularly attractive for streaming applications with timing constraints. Raptor codes [14] are based on LT codes [12] and are designed in order to achieve linear encoding-decoding time complexity.

Typically, Raptor codes concatenate weakened LT codes with outer codes (pre-coding step), as represented in Figure 2. In the LT coding stage, the source symbols are XOR-ed for generating the encoded symbols, and the implicit structure of the corresponding Tanner graph is passed to the decoder as a key along with the encoded symbols. The key, also called ESI, represent the seed of a pseudo-random generator determining both the encoding symbols degree, as well as the source symbols to be combined. One of the implementation of Raptor codes has been recently included in the 3GPP standard [2], where systematic Raptor codes employ a pre-coder consisting of regular LDPC codes and high density Half codes. The pre-coding stage is crucial to

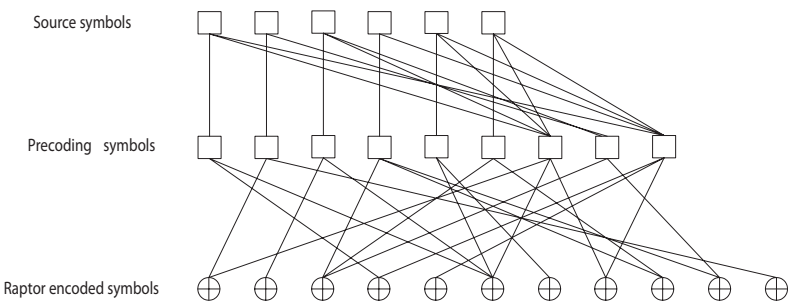

Figure 2: Encoding of non-systematic Raptor codes. The source symbols are first pre-coded and successively LT encoded.

enable low complexity encoding and decoding, as it permits the use of LT codes with sparse parity check matrices. Raptor codes have a very small overhead $\epsilon$ that tends to zero when the number of encoded symbols $K$ increases. In other words, the number of symbols $K(1+\epsilon)$ that needs to be received for correct decoding is close to $K$.

In more details, let $\mathbf{C}$ denote a source vector of $K$ input (source) symbols with $\mathbf{C}=\left[C_{1}^{T} C_{2}^{T} \ldots C_{K}^{T}\right]$. If we represent as $\mathbf{C}_{P}$ the redundant pre-coding symbols, the coding constraints among source and redundant symbols are given by

$$
\mathbf{C}_{P}=\mathbf{G}_{P} \cdot \mathbf{C}
$$

where $\mathbf{G}_{P}$ is the generator matrix of the pre-coder. The LT encoder then takes as input the pre-coding symbols $\mathbf{F}_{1: L}=$ $\left[\mathbf{C}^{T} \mathbf{C}_{P}^{T}\right]^{T}$ and generates the Raptor symbols, where $L=$ $K+S+H$ is the number of pre-coded symbols, and $S$ and $H$ are respectively the number of LDPC and Half codes constraints. If $\mathbf{G}_{L T}$ is the generator matrix of non-systematic LT encoder, then it holds that

$$
E_{1: N}=\left[E_{1}^{T} E_{2}^{T} \ldots E_{N}^{T}\right]^{T}=\mathbf{G}_{L T}(1,2, \ldots, N) \cdot \mathbf{F},
$$

where $N$ is the number of Raptor encoded symbols. The generator matrix of the LT coder $\mathbf{G}_{L T}$ is defined as

$$
\mathbf{G}_{L T}=\left[\boldsymbol{\Gamma}_{1}^{T} \boldsymbol{\Gamma}_{2}^{T} \ldots \boldsymbol{\Gamma}_{n}^{T}\right]
$$

where $\boldsymbol{\Gamma}_{i}^{T}$ with $i=1, \ldots, n$ is a row vector with ones at the positions corresponding to the indices of the pre-coded symbols that are combined for the generation of encoded symbols. Therefore, for every encoded symbol $i=1, \ldots, n$,

$$
E_{i}=\sum_{j \in \Psi_{i}} \bigoplus F_{j}
$$

where $\Psi_{i}$ is the set of pre-coding symbols indices that are combined and $\sum \bigoplus$ is the XOR operator. The $\Psi_{i}$ is determined by a pseudo-random generator whose seed is controlled by the ESI information.

The receiver performs Raptor decoding in order to recover the source symbols. It first constructs the matrix $\mathbf{A}$ with the encoding constraints given by the ESI's of the received symbols. The first rows of $\mathbf{A}$ contains the pre-coding conditions given by the generator matrices of the LDPC encoder, $\mathbf{G}_{L D P C}$ and of the Half encoder, $\mathbf{G}_{\text {Half }}$. It also includes the matrix $\mathbf{G}_{L T}^{\prime}$ that is a submatrix of $\mathbf{G}_{L T}$ where rows correspond to missing symbols that have been deleted. The matrix $\mathbf{A}$ can be written as

$$
\mathbf{A}=\left[\begin{array}{c}
\mathbf{G}_{L D P C} I_{S} O_{S \times H} \\
\mathbf{G}_{H a l f} I_{H} \\
\mathbf{G}_{L T}^{\prime}
\end{array}\right],
$$


where $I_{S}$ and $I_{H}$ are square unit matrices of size $S$ and $H$, respectively, and $O_{S \times H}$ is a zero matrix of size $S \times H$. If $\mathbf{A}$ has full rank, then the probability for successive decoding of the original source symbols becomes high. The decoder has to solve the following linear system of equations :

$$
\mathbf{A}_{[1 \ldots M]} \cdot \mathbf{C}=\mathbf{D}^{\prime}
$$

where $M>K$ is the number of received symbols. $D^{\prime}$ is a vector composed of a zero vector of length $S+H$ and the vector of received symbols. The above linear system is solved by Gauss-Jordan elimination.

\subsection{Raptor network coding}

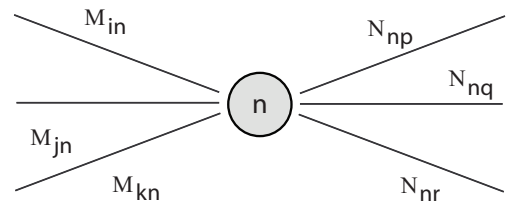

Figure 3: Raptor network coding peer.

$$
M_{n p}=\left(1-\pi_{n p}\right) N_{n p}
$$

We propose in this paper to apply Raptor coding in the network nodes, in order to improve the overall throughput of the streaming application. Network coding based on Raptor re-encoding in the network peers permits to avoid the computational cost and delays induced by decoding and encoding in the network nodes. It has small computational cost and keeps the probability of multiple packet reception very low. We use non-systematic Raptor codes as they offer linear encoding/decoding time and provides an effective symbol diversity for network coding.

Raptor coding is applied in overlay nodes, as illustrated in Figure 3. A node $n$ gathers coded symbols from ancestors nodes. It combines the input symbols $\left\{M_{i n}, M_{j n}, M_{k n}\right\}$ and transmits a subset $N_{n p}$ of the re-encoded symbols to the node $p$. The Raptor re-encoding matrix $\mathbf{A}^{\prime}$ is constructed using the ESI's of the received symbols. The Raptor reencoder generates new symbols combining some of the received symbols in order to compensate for losses. Note that the transmitted symbols can be erased before reaching the children peers, and we can write $M_{n p}=\left(1-\pi_{n p}\right) N_{n p}$.

The selection of symbols to be combined in network peer is crucial for high performance, since some symbol combinations can mislead the decoding process (i.e., the matrix $\mathbf{A}^{\prime}$ stays full rank, but the solution differs from the original). In order to avoid problems with the Gauss-Jordan elimination process at the receiver, we combine symbols that satisfy the following conditions : (a) they correspond to rows of the matrix $\mathbf{A}_{i}^{\prime}$ that are orthogonal to all rows of the original matrix $\mathbf{A}$ corresponding to erased symbols; (b) the rows $\mathbf{A}_{i}^{\prime}$ and $\mathbf{A}_{j}^{\prime}$ corresponding to combined symbols are orthogonal to each other. The first condition ensures that the new symbols are independent from the erased symbols. The second condition avoids the combination of non-orthogonal symbols, which can result in erroneous codewords. The Raptor re-encoding

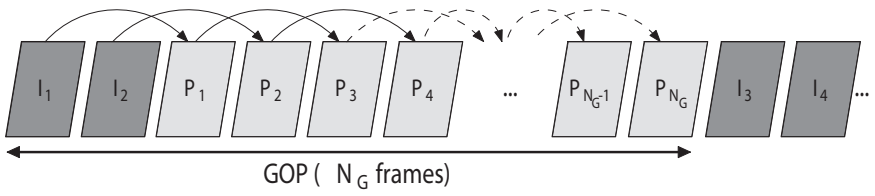

Figure 4: GOP structure of H264/AVC MDC scheme.

algorithm is applied as long as the previous conditions can be satisfied.

Obviously, the re-encoding strategy does not increase the rank of the matrix $A$, since the re-encoding symbols are combinations of received symbols. The performance of the Raptor codes therefore decreases when the number of hops augments, since the information conveyed by re-encoded symbols is also available in other encoded symbols. Symbol erasures and re-encoding make the matrix $A$ less sparse, so that Raptor re-encoding can only be applied a limited number of times. The network coding algorithm however takes advantage of the peer and path diversity. In particular, the rank of $A$ decreases more gracefully when multi-path transmission is combined with efficient packet splitting policy. In the proposed system, the sources and intermediate nodes split their packets into subsets of packets that are sent over different paths. The receiver can finally retrieve the source packets for any set of $K^{\prime}$ different packets with $K^{\prime}>K \cdot(1+\epsilon)$.

\section{RAPTOR VIDEO CODING}

We discuss in this section the application of the above network coding solution to video streaming applications. We first choose a non-systematic Raptor coder based on the encoder defined in the 3GPP standard [2]. Raptor codes generally perform satisfactory when the number of source symbols is sufficiently large and the overhead of the codes is kept as small as possible. However, very small packets are not appropriate for multihop transmission due to the overhead added by the transport protocols (RTP/UDP/IP overhead is around 40 bytes). We use packets of 512 bytes, as proposed in the $3 \mathrm{GPP}$ standard. If the overall bitrate is very low, several symbols are fed together in the same packet. A 2-byte ESI information field is finally appended to each packet with a negligible overhead.

Then, the number of encoded symbols has to be sufficiently high for effective performance of the Raptor coding system, since it is linked to the number of source symbols $K$. We therefore build independent groups of symbols for each Group of Pictures (GOP) in the bitstream, which represents the pictures between two reference frames. At the same time, the GOP size is limited by latency constraints. For transmission of CIF sequences coded at $30 \mathrm{fps}$, we typically select a GOP size of 60 frames.

Since we use a non-systematic code, the efficiency of error concealment at the decoder is very limited in case of decoding failure. We therefore propose to improve the robustness to error with a simple Multiple Description Coding (MDC) scheme. The video sequence is split into two independent encoding threads, as illustrated in Fig. 4. The odd $P$ frames use the first $I$ frame as reference frame, while the even $P$ frames refer to the second $I$ frame. The MDC coder enhances system robustness because the simultaneous 
loss of both descriptions is quite improbable. Whenever the decoder fails to retrieve the source information from one description, the other description is used for error concealment with simple frame replication.

\section{SIMULATION RESULTS}

We now analyze the performance of the proposed network coding algorithm in regular and irregular overlay topologies, similar to the one illustrated in Figure 1. The source nodes transmit video streams encoded at the same code rate using the MDC video coder of Section 3. The proposed MDC video coder is based on JM 12.2 [1] of the H.264/AVC standard. Half pixel motion compensation is used and the reference frame buffer value is limited to one. The video encoder assigns one packet per NALU. Losses are generated based on a Gilbert-Elliott model, which is a two-state Markov chain whose transition probabilities drive the packet loss ratio and the average error burst length.

The proposed Raptor network coding scheme is compared with a baseline scheme performing Raptor decoding and encoding in every intermediate node. The decoding and encoding scheme replicate randomly the received packet, whenever Raptor decoding fails or when the number of encoded packets is not sufficient to fill the outgoing bandwidth. Alternatively, the network coding performs symbol re-encoding in the peers, in order to replace erased packets and meet the output bandwidth link. Whenever there are not enough packets to hit the outgoing bandwidth capacity with network coding, it randomly replicates some of the incoming packets to fill the bandwidth. When the outgoing bandwidth is insufficient, both schemes randomly select a subset of packets to be transmitted. No reconciliation is performed between network nodes. Finally, all results have been averaged on 100 simulation runs.

We first analyze the evolution of the performance of the Raptor network coding algorithm, with the number of hops between the encoder and the decoder as illustrated in Figure 5. Simulations have been performed for a regular topology with 3 peers per hop. We assume that each link has a capacity of $300 \mathrm{kbps}$, and 158 packets of 512 bytes each have been generated with 140 video packets. We observe that the performance of Raptor re-encoding decreases with the number of hops, as expected. In addition, the re-encoding strategy is mostly beneficial at high average loss rate, when compared to encoding and decoding in the network nodes. The results in Figure 6 confirms these observations, as they show that the probability of failure decreases more rapidly in the re-encoding strategy, when the number of received symbols increases. This is due to the high number of packet replicates in the decoding and encoding strategy.

Then, we analyze the behavior of the network coding strategy as a function of the average link bandwidth, $\bar{r}$. The average packet loss ratio is set to 0.05 , and the link bandwidth is randomly selected in $[\bar{r}-40, \bar{r}+40] \mathrm{kbps}$. The "Foreman" CIF sequence is encoded at $512 \mathrm{Kbps}$, and the Raptor symbol size is 512 bytes. We compare in Figure 7 the performance of the network coding and the baseline schemes for a regular $(2-3-3-2)$ and an irregular $(2-2-3-2)$ overlay network topology. From Figure 7(a) it can be seen that the decoding and encoding approach performs better than the Raptor network coding approach for a regular $2-3-3-2$ topology with sufficient diversity. The performance gap is quite significant for low average link bandwidth. The per-

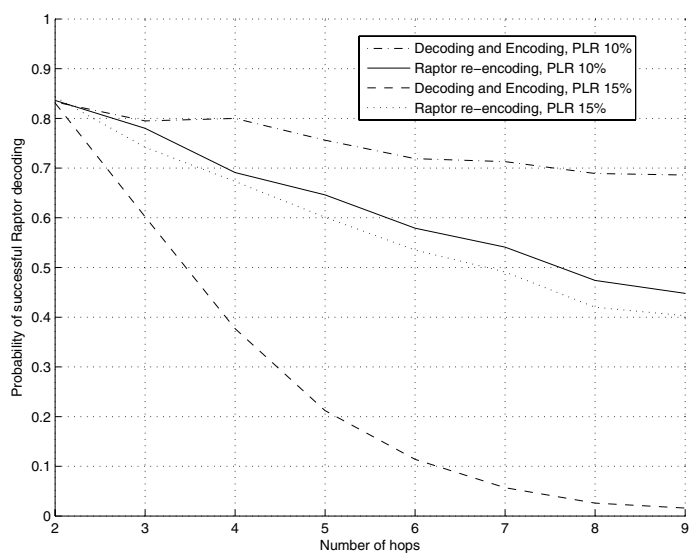

Figure 5: Probability of decoding vs number of stages/hops.

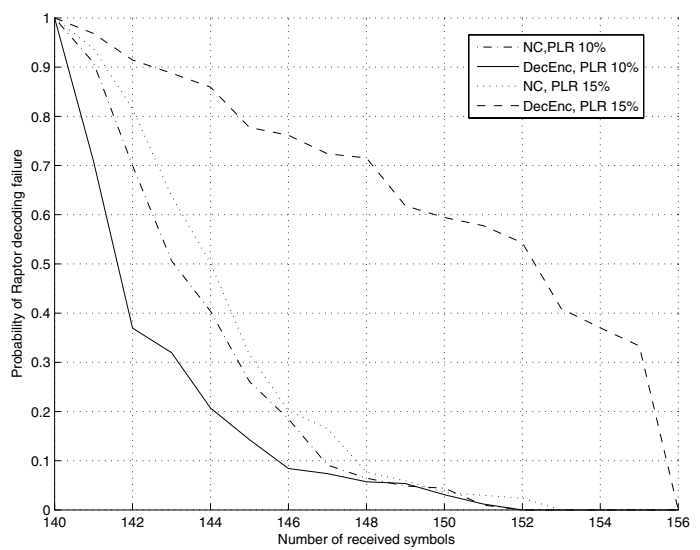

Figure 6: Probability of decoding failure vs number of symbols, in a 5-hop topology.

formance difference then decreases sharply as the average link capacity increases, and both methods perform similarly when the bandwidth is high. The improved performance of the decoding and encoding approach is due to the replication policy followed in the intermediate nodes. It ensures successful decoding at the second hop nodes and the receivers. On contrary, the Raptor network coding scheme cannot maintain a sufficiently high packet diversity for low bandwidth links. It hardly succeeds in exploiting path diversity to compensate for the decay of the packet diversity, which reduces the probability that the end-nodes receive a set of packets that corresponds to full rank generator matrix.

The influence of the link capacity is more apparent when we evaluate the coding scheme in a irregular $2-2-3-2$ topology, as shown in Figure 7(b). Since the first hop has only two nodes, the packet replication policy can not assist decoding and encoding scheme since the set of packets that arrive at the second hop does not correspond to a linear equation system with full rank. For this topology, the network coding system even performs best, since it becomes less sensitive to the above problems. However, it should be noted that the system needs slightly higher average bandwidth to guarantee error free video decoding, when compared to the regular topology. 
Finally, we compare the performance of both schemes with the link bandwidth is quite heterogeneous. The average link capacity $\bar{r}$ is set to 250 and $300 \mathrm{kbps}$ respectively for the regular $2-3-3-2$ and the irregular $2-2-3-2$ topologies, respectively. The actual link bandwidths are then randomly selected as $\bar{r}+\sigma$, with $\sigma \in[20,100]$ kbps. The results confirm the previous analysis, as the the decoding and re-encoding strategy performs best for the regular topology (see Figure 8(a)), due to the large variations of bandwidth. For the irregular topology, the performance are again better for the network coding algorithm, as the baseline scheme fails to transmit enough different packets due to the reduced number of peers (see Figure 8(b)). Overall, the simulation results highlights the importance of the average link capacity for good performance of streaming solutions based on network diversity. Proper rate allocation therefore becomes critical, since the network coding algorithm performs close to decoding and re-encoding strategies for well-defined channel conditions, but outperforms it when channel characteristics are not correctly estimated.

\section{RELATED WORK}

Network coding has received a lot of attention from the research community in the past few years. Network coding schemes that have been proposed in the literature can be classified in two major categories, namely the systems based on algebraic and channel coding. An algebraic perspective on network coding has first been provided in [9], which shows that maximal robustness to non-ergodic link failures can be achieved. Linear network codes have been considered in [10] for multi-hop networks, where they achieve the max-flow capacity bound with controllable complexity in the network nodes. Recently, the benefits of network coding has been thoroughly examined in wireless sensor networks with dynamically changing topologies [5]. Alternatively, channel codes have also been proposed for network coding. For example, a network coding system based on LDPC codes has been proposed in [4] for transmission over wireless relay networks. Joint network-channel coding (JNCC) has been proposed recently for transmission over relay channels [7], based on Turbo codes. In this paper, we have proposed to use Raptor codes for network coding, in order to take benefit from their rateless and low complexity properties in realtime streaming systems.

In addition, a few works have addressed lately the application of network coding principles to streaming applications. Reed-Solomon codes or LT codes have been implemented in network-embedded FEC nodes [18] and in network peers [17], respectively, in order to enhance the robustness to transmission errors. In both cases, the packets are decoded and re-encoded in the network nodes, before transmission towards the streaming client. Both schemes show that the network throughput can be significantly improved with network coding. However, decoding and recoding operations in network nodes augments the latency of the streaming system. Network coding has also been proposed for multicasting in overlay or peer-to-peer networks $[19,11,6,8]$, where it takes advantage of path diversity. The benefit of network coding with peer-to-peer networks has been evaluated in [15], which shows that network coding is very useful in peer-to-peer networks, since it provides granularity and resiliency to network dynamics and leads to better bandwidth usage.

\section{CONCLUSIONS}

We have proposed in this paper a video network coding algorithm based on Raptor codes for media streaming in lossy packet networks. Information symbols are re-encoded in the network nodes, before transmission to the next hop towards the media client. Re-encoding permits to avoid the complexity and delay due to decoding and re-encoding operations implemented in state-of-the-art solutions. Such a network coding solution shows to be beneficial at high loss rate, or when the network diversity is limited. The results also illustrate that efficient rate allocation is certainly crucial for the deployment of effective network coding systems in heterogeneous networks such as wireless mesh networks.

\section{REFERENCES}

[1] Jvt reference software version 12.2:. [Online], Available on web site http://bs.hhi.de/ suehring/tml/.

[2] 3GPP TS 26.346 V7.1.0, Technical Specification Group Services and System Aspects; Multimedia Broadcast/Multicast Service; Protocols and Codecs, June 2005.

[3] R. Ahlswede, N. Cai, S.-Y. R. Li, and R. W. Yeung. Network Information Flow. IEEE Trans. Information Theory, 46(4):1204-1216, Jul. 2000.

[4] X. Bao and J. Li. A Unified Channel-Network Coding Treatment for Wireless Ad-Hoc Networks. In Proc. of IEEE International Symposium on Information Theory, ISIT 2006, Seattle, WA, USA, July 2006.

[5] C. Fragouli, J. Widmer, and J. Y. L. Boudec. A Network Coding Approach to Energy Efficient Broadcasting: from Theory to Practice. In Proc IEEE Infocom 2006, Barcelona, Spain, Apr. 2006.

[6] C. Gkantsidis, J. Miller, and P. Rodriguez. Comprehensive View of a Live Network Coding P2P system. In Proc ACM SIGCOMM/USENIX IMC'06, Brasil, Oct. 2006.

[7] C. Hausl and P. Durpaz. Joint Network-Channel Coding for the Multiple-Access Relay Channel. In Proc. International Workshop on Wireless Ad Hoc and Sensor Networks, New York, USA, June 2006.

[8] K. Jain, L. Lovász, and P. A. Chou. Building Scalable and Robust Peer-to-Peer Overlay Networks for Broadcasting Using Network Coding. Journal on Distributed Computing, 19(4):301-311, Dec. 2006.

[9] R. Koetter and M. Médard. An Algebraic Approach to Network Coding. IEEE/ACM Trans. on Networking, 11(5):782-795, Oct. 2003.

[10] S.-Y. R. Li, R. W. Yeung, and N. Cai. Linear Network Coding. IEEE Trans. Information Theory, 49(2):371-381, Feb. 2003.

[11] Y. Liu, Y. Peng, W. Dou, and B. Guo. Network Coding for Peer-to-Peer Live Media Streaming. In Proc. Fifth International Conference Grid and Cooperative Computing, GCC 2006, pages 149-155, Monticello, IL, USA, Oct. 2006.

[12] M. Luby. LT codes. In Proc. of the 43rd Annual IEEE Symposium on Foundations of Computer Science (FOCS '02), pages 271-280, Vancouver, Canada, Nov. 2002.

[13] M. Luby, M. Watson, T. Gasiba, T. Stockhammer, and W. Xu. Raptor Codes for Reliable Download Delivery in Wireless Broadcast Systems. In Proc. of 


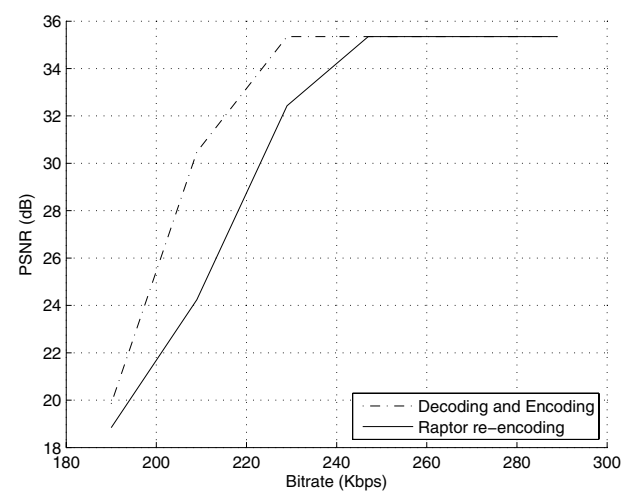

(a)

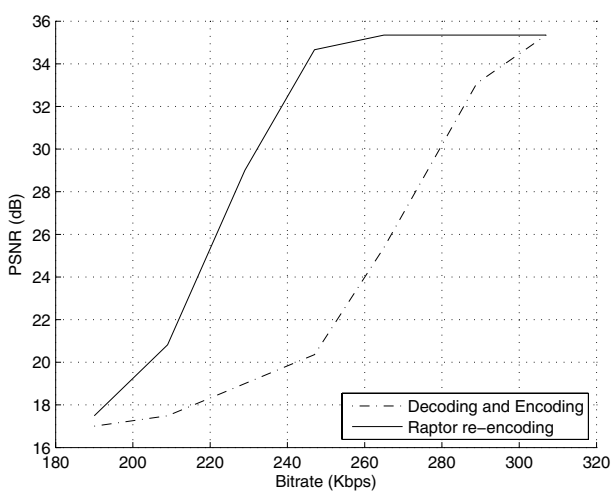

(b)

Figure 7: PSNR comparison for transmission of the "Foreman" CIF sequence coded at 512 Kbps with respect to average link capacity for (a) $2-3-3-2$ and (b) $2-2-3-2$ overlay network topologies.

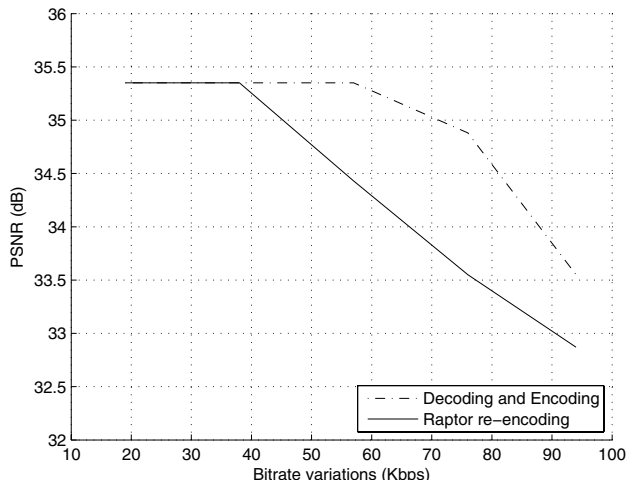

(a)

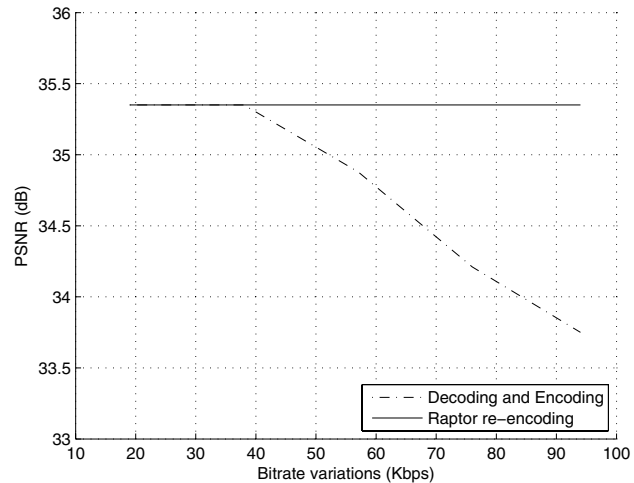

(b)

Figure 8: PSNR comparison for transmission of the "Foreman" CIF sequence coded at $512 \mathrm{Kbps}$ with respect to the variation at the average link capacity for (a) $2-3-3-2$ and (b) $2-2-3-2$ overlay network topologies.

3rd IEEE Consumer Communications and Networking Conference, CCNC2006, volume 1, pages 192-197, Jan. 2006.

[14] A. Shokrollahi. Raptor codes. IEEE Trans. Information Theory, 52(6):2551-2567, June 2006.

[15] M. Wang and B. Li. Lava: A Reality Check of Network Coding in Peer-to-Peer Live Streaming. In Proc. of IEEE INFOCOM, Anchorage, Alaska, May 2007.

[16] C. Wu and B. Li. rStream: Resilient and Optimal Peer-to-Peer Streaming with Rateless Codes. IEEE Trans. on Parallel and Distributed Systems. Accepted for publication.
[17] C. Wu and B. Li. Optimal Peer Selection for Minimum-Delay Peer-to-Peer Streaming with Rateless Codes. In Proc. of ACM Workshop on Advances in Peer-to-Peer Multimedia Streaming, P2PMMS 2005, pages 69-78, Singapore, Nov. 2005.

[18] M. Wu, S. Karande, and H. Radha. Network Embedded FEC for Optimum Throughput of Multicast Packet Video. EURASIP Journal on Applied Signal Processing, 20(8):728-742, Sep. 2005.

[19] J. Zhao, F. Yang, Q. Zhang, Z. Zhang, and F. Zhang. LION: Layered Overlay Multicast With Network Coding. IEEE Trans. Multimedia, 8(5):1021-1032, Oct. 2006. 